\title{
The effect of liver transplantation for argininemia - the largest experiences in a single center
}

\author{
Bin Cui ${ }^{1,2,3}$, Lin Wei ${ }^{1,2,3}$, Li-Ying Sun ${ }^{1,2,3,4}$, Wei Qu ${ }^{1,2,3}$, Zhi-Gui Zeng ${ }^{1,2,3}$, Ying Liu ${ }^{1,2,3}$, Zhi-Jun Zhu ${ }^{1,2,3}$ \\ ${ }^{1}$ Liver Transplantation Center, Beijing Friendship Hospital, Capital Medical University, Beijing, China; ${ }^{2}$ Clinical Center for Pediatric Liver \\ Transplantation, Capital Medical University, Beijing, China; ${ }^{3}$ National Clinical Research Center for Digestive Diseases, Beijing, China; ${ }^{4}$ Department \\ of Critical Liver Diseases, Liver Research Center, Beijing Friendship Hospital, Capital Medical University, Beijing, China \\ Contributions: (I) Conception and design: ZJ Zhu, LY Sun, L Wei; (II) Administrative support: All authors; (III) Provision of study materials or \\ patients: W Qu, ZG Zeng, B Cui, Y Liu; (IV) Collection and assembly of data: W Qu, ZG Zeng, B Cui, Y Liu; (V) Data analysis and interpretation: \\ B Cui; (VI) Manuscript writing: All authors; (VII) Final approval of manuscript: All authors. \\ Correspondence to: Zhi-Jun Zhu. No. 101 Lu Yuan Dong Road, Tong-Zhou District, Beijing 101100, China. Email: zhu-zhijun@outlook.com.
}

Background: Argininemia, a rare urea cycle disorder resulting from an arginase-1 deficiency, is characterized by a progressive spastic paraplegia. While advances in diagnosis and treatment have increased the management of this condition, not all symptoms are resolved in response to traditional therapies. Interestingly, there exist some rare reports on the use of liver transplantation (LT) for the treatment of argininemia.

Methods: We conducted a retrospective study of eleven patients with argininemia receiving a LT as performed at our center over the period from January 2015 to November 2019. These patients were included due to their poor responses to protein restriction diets and alternative therapies of nitrogen scavengers. Detailed information on coagulation, liver function, histopathological and morphological examination of liver samples, and other clinical presentations were extracted from these patients. A grading scale was used for evaluating the neurological status, classification of physical growth and quality of life of these patients in response to the LT.

Results: Prior to LT, high levels of arginine were detected in all of argininemia patients and liver enzymes were elevated in nine of those patients. Nine patients presented with coagulation dysfunction without bleeding symptoms. Spastic paraplegia, irritability, intellectual developmental disability, and growth deficits were hallmarks of these nine patients, while four patients showed repeated, generalized tonic-clonic seizures before the operation. Seven novel mutations were found in these patients. The indication for LT in this series of patients was a presentation of progressive neurological impairments. After LT, the coagulation index and plasma arginine levels returned to normal and episodes of seizure were controlled in four patients. To date, all patients have survived and their LT has resulted a restoration of arginine metabolism and liver function, along with preventing further neurological deterioration, all of which provided an opportunity for future recuperation. Overall, the neurological status, growth deficits and quality of life were all significantly improved after LT with no evidence of severe complications.

Conclusions: LT can serve as an effective treatment for argininemia in patients who respond poorly to traditional therapy. An early intervention of LT should be conducted in these patients to prevent neurological damage and improve their quality of life.

Keywords: Argininemia; liver transplantation (LT); urea cycle disorders (UCDs); neurological damage

Submitted Dec 06, 2021. Accepted for publication Jan 27, 2022.

doi: $10.21037 /$ tp-21-576

View this article at: https://dx.doi.org/10.21037/tp-21-576 


\section{Introduction}

Argininemia (arginase deficiency or hyperargininemia; OMIM 207800) is an autosomal recessive inherited disease caused by a deficiency of arginase-1 (ARG1) (1). The basis for this genetic disorder has been traced to inborn mutations in the ARG1 gene located on chromosome 6q23.2. As a result, there is a partial or complete loss in the function of this enzyme. Some 70+ mutations have been reported as being dispersed throughout the $A R G 1$ gene (2). Argininemia is pan-ethnic, with an estimated incidence of 1 in 2 million live births $(1,3,4)$. The accumulation of arginine within the blood and other fluids represents the hallmark of argininemia and is present in all patients.

As compared with other urea cycle disorders (UCDs), the clinical presentations of argininemia are complicated and lack specificity, including progressive spastic paraplegia, seizures, growth deficits, intellectual developmental disability, microcephaly, irritability, lethargy, nausea, reduced appetite, and ataxia, while hyperammonemic encephalopathy is rarely observed in argininemia $(3,4)$. In addition to neurological damage, liver damage ranging from a mild elevation of transaminases to liver failure is associated with argininemia (5). Coagulation dysfunction is also a characteristic of argininemia, however, this symptom is not accompanied with life-threatening hemorrhagic complications and the underlying mechanisms remain unclear (6).

Treatment of argininemia should start with a combination of protein restriction diet along with supplements of essential amino acids, sodium benzoate and sodium phenylbutyrate. However, responses to these conservative treatments vary among patients and may be ineffective in some cases. For example, neurological symptoms could not be entirely prevented by conventional treatments (6), as some of the neurotoxic guanidine metabolites of arginine can remain elevated (7). Interestingly, plasma levels of arginine and its metabolites have been reported to be normalized by liver transplantation (LT), which can then significantly alleviate these neurological symptoms (7). However, the effects of LT as used in the treatment of argininemia have rarely been reported.

Given the potential benefits, but rare reported evidence, for use of LT in patients with argininemia, detailed descriptions of individualized treatment plans and clinical presentations may provide important information and unique insights, not only for the understanding of the underlying physiopathological mechanisms but also for timely interventions in such cases. Therefore, in this report we describe the characteristics and the clinical outcomes of eleven patients with argininemia receiving LT in our center. We present the following article in accordance with the STROBE reporting checklist (available at https:// tp.amegroups.com/article/view/10.21037/tp-21-576/rc).

\section{Methods}

\section{Study population}

A retrospective analysis of eleven patients (seven female and four male) with argininemia was performed. These patients were admitted to the Liver Transplantation Center of the Beijing Friendship Hospital, Capital Medical University over the period between January 2015 and November 2019. Blood arginine levels were analyzed by liquid chromatography tandem mass spectrometry method, and gene sequencing was performed in all patients to confirm mutations in $A R G 1$. Indications for LT were progressive neurological impairment and metabolic decompensation following traditional treatments (8). Transplantation scores were assessed according to that as described in a previous report (9) and all LT surgeries performed were approved by the Ethics Committee of Beijing Friendship Hospital. Preand post-surgical data on coagulation, liver function and clinical presentations were collected to evaluate the effects of LT. A grading scale, as based on that of Morioka et al. (10), was used to assess the neurological status, physical growth and quality of life of the patients who were followed up at the clinic.

\section{Statistical analysis}

Data are presented as mean \pm standard deviation (SD). The small number of participants in this study precluded use of formal statistical analyses.

\section{Ethical statement}

The study was conducted in accordance with the Declaration of Helsinki (as revised in 2013). This study was approved by the Ethics Committee of Beijing Friendship Hospital, Capital Medical University (No. 2020-P2094-01). Informed consents were obtained from all legal guardians of participants included in the study. 


\section{Results}

\section{Preoperative clinical characteristics}

Genetic analysis revealed two allele mutations in the $A R G 1$ gene within all eleven patients. These mutations were derived from their parents and were consistent with autosomal recessive inheritance characteristics. Among the eleven patients, sixteen mutations were identified, with seven mutations, c. $23 \mathrm{~T}>\mathrm{C}$ in exon 1 , c.51delG in exon 1, c.246_248delAAA in exon 3, c.603delT in exon 6 , c.756-757insACAT in exon 7, c. $826+2 \mathrm{~T}>\mathrm{C}$ in exon 7 , and c. $922 \mathrm{C}>\mathrm{T}$ in exon 8 were novel. Three patients were found by neonatal screening (Cases 3, 4, and 6), and confirmed by gene sequencing. The remaining eight patients were diagnosed by plasma amino acid analysis and gene sequencing after symptoms had developed. The average age at onset of argininemia was $18.8 \pm 14.3$ months (range, 4-54 months). A summary of the main preoperative characteristics of the patients are contained in Table 1.

Nine patients presented with typical spastic paraplegia of the lower extremities and two patients (Cases 4 and 9) manifested ataxic tremors of the upper extremities. Results from physical examinations revealed hypermyotonia, bilateral muscular weakness within the lower limbs, hyperreflexia of knee and ankle reflexes, positive ankle clonus and Babinski signs. Two patients (Cases 3 and 6) failed to show any obvious dyskinesia, which might be attributable to their younger age. Four patients (Cases $3,6,9$, and 10) had generalized tonic-clonic seizures as their initial symptoms, with their electroencephalograms showing continuous generalized spikes or polyspike/spikeslow complex wave discharge (Figure S1A). Topiramate and levetiracetam were used to control their seizures. Five patients (Cases 1, 8, 9, 10, and 11) were incapable of walking independently and required help during the preoperative evaluation while the remaining cases showed a "tip-toe" gait and could only walk for short distances. Neuroimaging revealed severe atrophy of the cerebellum and brainstem, with an enlarged fourth ventricle (Figure S1B). Cases 8 and 9 were initially misdiagnosed with cerebral palsy (CP) and received rehabilitation training, but their conditions continued to deteriorate. Only Case 7 was capable of slow speech, while all other patients lost the ability to speak sentences and showed intellectual developmental disability. Growth deficits were observed in seven patients and all patients manifested emotional instability and irritability. Hyperammonemic encephalopathy was not present in any of the patients in this series. Data on neurological status, classification of physical growth and quality of life are contained in Table 2.

\section{Preoperative laboratory data and liver imaging}

The range of maximal blood arginine concentrations was $187-810 \mu \mathrm{mol} / \mathrm{L}$, with an average of $459 \pm 209 \mu \mathrm{mol} /$ L. Nine patients had elevated liver transaminases, while no substantial changes were observed in albumin and total bilirubin, and platelet counts were normal. Results from coagulation analysis indicated that prothrombin time (PT) was prolonged, international normalized ratio (INR) was increased in nine patients and activated partial thromboplastin time (APTT) was markedly prolonged $(>10 \mathrm{~s})$ in five patients. None of the patients presented with significant bleeding symptoms and, as a clotting factor assay were not routinely conducted as part of the preoperative evaluation, no further assessments of coagulation factor deficiencies were performed in this group. During the perioperative period, mild hyperammonemia was found in the blood sample of ten patients. These data are listed in Table 3. Abdominal computed tomography showed mild hepatomegaly in two patients (Cases 2 and 9) and results of an ultrasound examination revealed an enhancement of liver echogenicity in four patients (Cases 3, 6, 8, and 9). These laboratory data were collected during the evaluation for LT while all patients were on a protein-restricted diet. A range of blood ammonia and arginine concentration were collected in some cases.

\section{Postoperative LT data}

All patients seek LT because their progressive neurological damage after conventional treatment. The mean transplantation score was $11.0 \pm 1.5$, which an absolute indication for LT (9). Of these eleven patients, seven received living donor liver transplantation (LDLT), the other four received deceased donor liver transplantation (DDLT). All patients underwent LT by a standard procedure $(11,12)$. The average age at LT was $48.3 \pm 43.3$ months old. The mean value of graft-to-recipient weight ratio (GRWR) was $2.5 \%$ (range, $1.3-3.8 \%$ ). Within the LDLT group, donors were heterozygote. Immunosuppressive treatment consisted of tacrolimus, mycophenolate mofetil and low doses of corticosteroids. Postoperative complications included biliary stricture in Case 1, portal vein stenosis in Case 3 and no major complications in the remaining patients. The postoperative follow-up period ranged from 


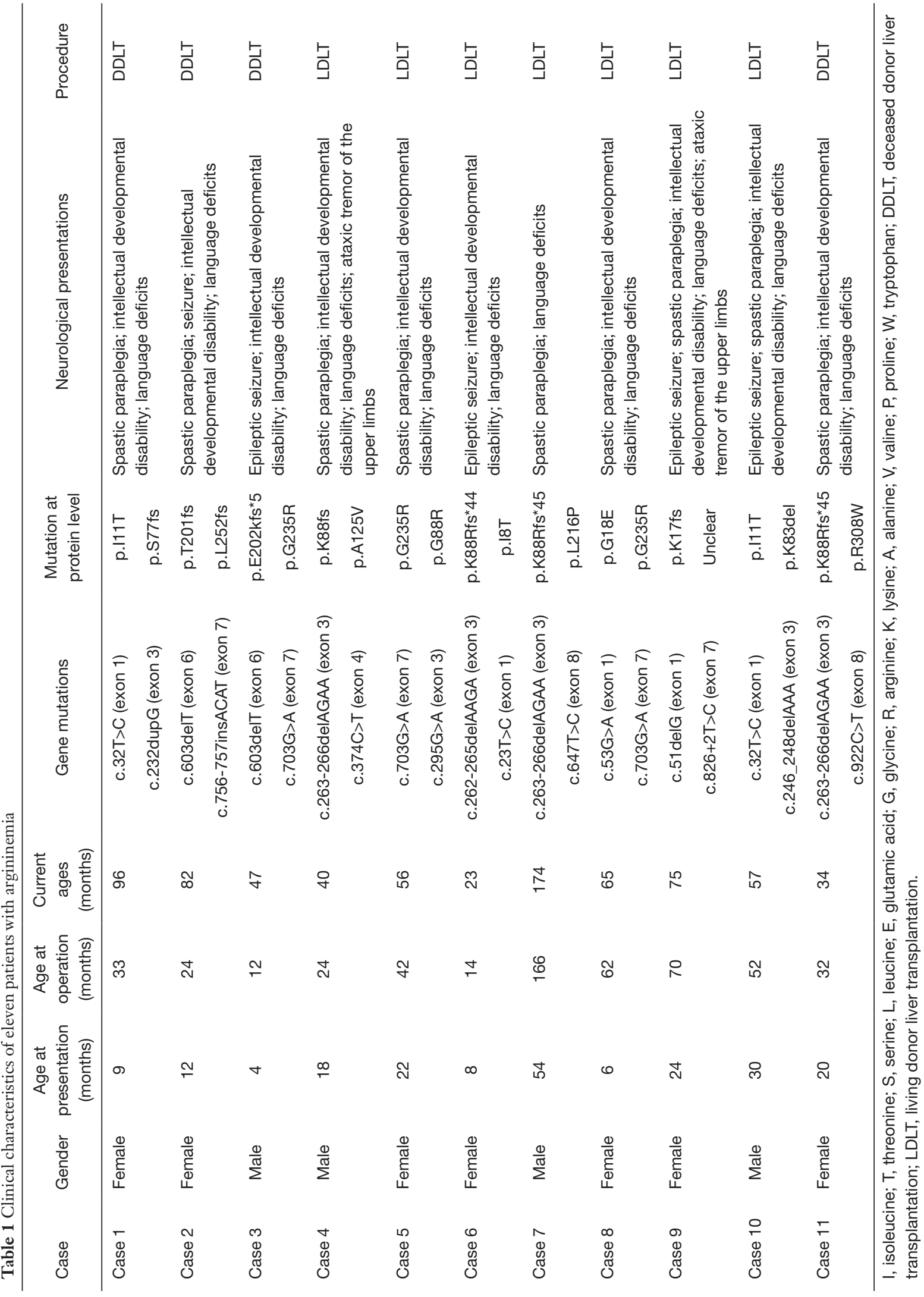




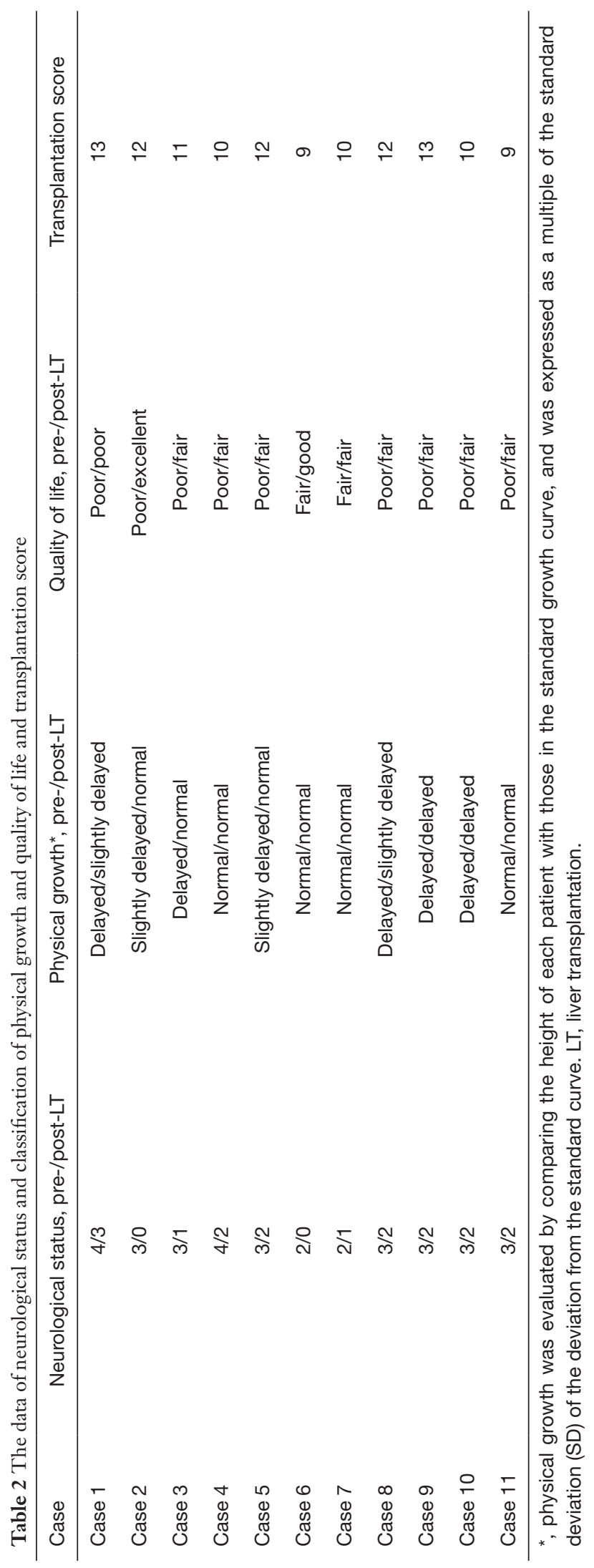

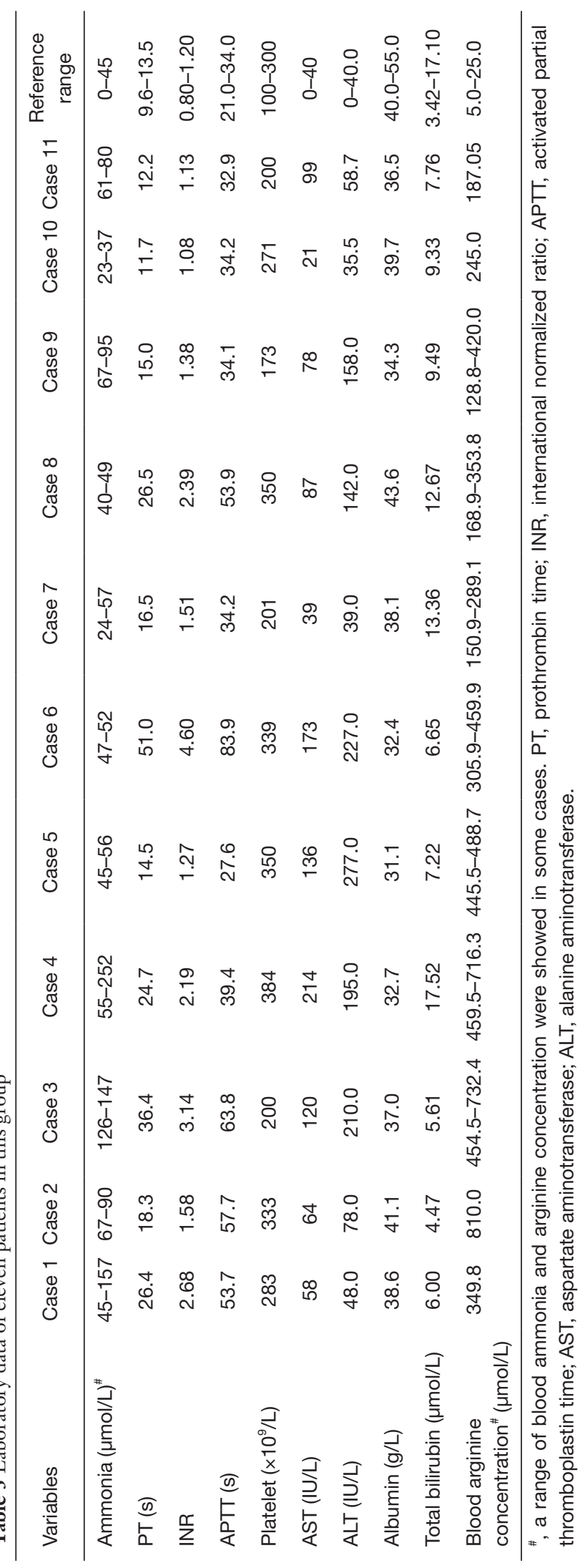



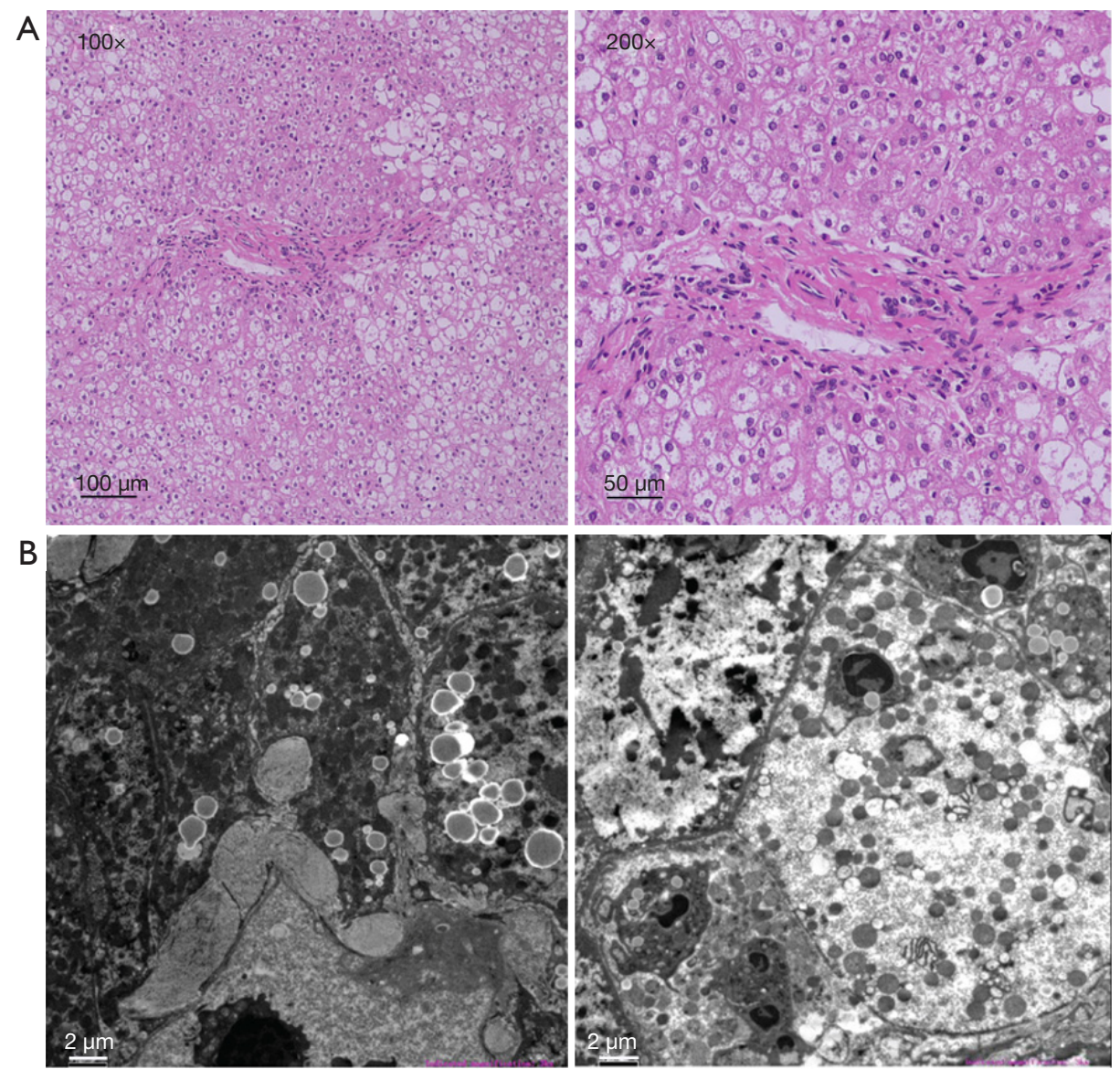

Figure 1 Pathological characteristics within the liver of argininemia patients. (A) Hematoxylin-eosin (HE) staining showing swollen "ballooned" hepatocytes, patchy fatty vacuolars, lymphocytes infiltration within portal areas. (B) Electron microscopy showing damaged hepatocytes with substantial swelling, an increased number of mitochondria with unclear ultrastructures and diffuse lipid droplets, while the shape and disposition of the endoplasmic reticulum appeared normal.

2 to 63 months. To date, overall patient and graft survival rates are $100 \%$.

Hematoxylin-eosin (HE) staining showed swollen hepatocytes, lymphocytes infiltration, and large patchy fatty vacuolars in hepatocytes. These swollen "ballooned" cells contained normal-sized central nuclei (Figure 1A). None of the patients manifested liver cirrhosis. Swollen hepatocytes containing varying sizes of lipid droplets with enlarged gaps were observed with electron microscopy. The number of mitochondria increased and their ultrastructure was indistinct. The endoplasmic reticulum appeared normal and a large number of collagen fibers were deposited between hepatocytes and in the Disse space (Figure 1B).

Plasma ammonia concentrations and coagulation dysfunction returned to normal within one month after the operation, even after resumption of their normal diet. Arginine metabolism was also normal after LT, as evidenced by decreases in plasma arginine concentrations and normalized orotic acid levels in the urine. All patients discontinued their dietary restrictions after LT without protein aversion and any metabolic decompensation.

All patients were emotionally stable, and their irritable state completely dissipated after LT. Catch-up growth was observed in seven patients affected by short stature, with three of these patients achieving a normal height, which may be attributable to the promotion of arginase and an enhanced nutritional status (Table 2). In this study, Cases 7 to 11 showed a substantial decrease in muscle tone with a slight improvement in their gait shortly after LT (Video S1). Patient 2 was now able to walk without any abnormalities, while patients 1, 4, and 5 continued to show a "tip-toe" gait. Knee-jerk reflexes of patients affected by spastic paraplegia 
returned to normal in all patients and the hypermyotonia within the lower extremity improved significantly at rest. However, only in Case 2 was there a complete recovery of gastrocnemii. As of their last assessment, four patients that had been affected by epilepsy remained seizure free, without any antiepileptic drugs. Patients 2, 7 and 6 were able to communicate normally after LT, while the remaining patients with preoperative language deficits could now speak in simple sentences. Patient 2 was enrolled in school and exhibited ageappropriate social skills and psychomotor abilities, with no apparent learning disabilities. Patient 6 recovered entirely and is not yet of school age. The other patients affected by intellectual developmental disability could now respond to simple spoken requests from their parents and became excited in the presence of their family or familiar friends. Although not all symptoms were alleviated completely, both their neurological status and growth deficits showed substantial improvements. Moreover, the quality of life improved in ten patients when assessed in their short-term follow-up. Of course, the 2-3 immunosuppression drugs used in Cases 4-11 within the first year post-LT affected the quantitative value of life quality assessment scores. These data are contained in Table 2.

\section{Discussion}

ARG was discovered within mammalian liver tissue in 1904. The two isoenzymes related to ARG, ARG1 and ARG2, were found to be encoded by different genes in mammals $(13,14)$, with ARG1 being expressed in highest levels within the cytosol of liver cells while ARG2 was mainly expressed in the prostate and kidneys. ARG1 was the final enzyme of the urea cycle, converting arginine to urea and ornithine (14). A deficiency of ARG1 can result in impaired ureagenesis, which is characterized by hyperargininemia (3). The ARG1 gene was located on chromosome 6 (6q23.2) and comprised eight exons, and mutations in this gene cause alterations in the structure of ARG1 thereby preventing it from functioning correctly (15). Although the functions of these two enzymes can be somewhat different, a functional overlap between them may explain why severe hyperammonemia was absent in patients with argininemia $(5,16)$.

The neurophysiological evaluation data of Cases 7 and 11 showed that the central nervous system was affected, while peripheral motor conduction time, somatosensory evoked potential, electromyograph and nerve conduction velocity were all within normal ranges, suggesting that a corticospinal tract impairment was involved in the pathophysiological mechanism (17). The latest research on argininemia indicates that it causes dysmyelination in the white matter of the brain, suggesting that the pathogenesis of argininemia is related to a decrease in oligodendrocytes (18). In the arginase deficiency model of Caenorbabditis elegans an accumulation of arginine has been shown to disrupt mitochondrial homeostasis. At the same time, a genetic inactivation of the mitochondrial basic amino acid transporter SLC-25A29 could rescue the mitochondrial defects, meaning energy metabolism impairment may be the reason for the damage of the central nervous system (19). In support of this proposal are the findings that nitric oxide (NO) induces demyelination by impairing the energy metabolism of oligodendrocytes (20). Lesions of the corticospinal tract resulting from disorders in oligodendrocyte energy metabolism may be responsible for the neurological damage. Our electron microscopy data suggested that mitochondrial damage resulting from arginase deficiency may be responsible for the liver damage. We speculated that the impairment observed within the central nervous system might also be caused by a mitochondrial dysfunction, a possibility which will require further experimental verification.

Arginase deficiency is the rare kind of UCDs. While the pathological mechanisms of argininemia resulting in neurological damage is still unknown, they may be related to a direct effect of arginine or its metabolites, such as guanidino compounds and NO (5). Guanidino compounds may damage the brain via oxidative stress (21). One research has reported that guanidinoacetate is a therapeutic target and biomarker for therapeutic monitoring in argininemia, but the accuracy of this agent has yet to entirely elucidated (22). Plasma metabolomic data from 13 argininemia patients have showed that elevated levels of guanidino compounds, guanidinoacetate, argininate, 2-oxoarginine, and $\mathrm{N}$-acetylarginine may contribute to the phenotype (23). Although conservative treatments for argininemia, including protein restriction, pharmacologic nitrogen scavenger therapy, and symptomatic and supportive protocols, can efficiently control primary manifestations and secondary complications in some patients, as many as $25 \%$ of patients show a progressive exacerbation $(4,7)$. At present, the only alternative treatment available to prevent neurological damage is LT, which could normalize the arginine levels and its metabolites to average ranges (7). Historically, the risks of morbidity and mortality as associated with LT and the need for long-term immunosuppression have often led to 
a preference for medical management. However, pediatric LT recipients could get an excellent long-term survival along with the improvement in both surgical technique and immunosuppression management (24). Here, we report clear beneficial effects of LT on argininemia as observed within a relatively short-term follow-up period. As different scales have been used in assessing patients in previous reports, in this report we used a single grading scale to evaluate neurological status, classification of physical growth and quality of life to record the preoperative and postoperative data in these patients (10). We found most patients were benefited from the operation. All patients and grafts survived, as determined at the end of the follow-up period, and showed an improved quality of life along with a regular dietary intake. Only two cases were affected with mild complications.

Neurological damage is difficult to restore due to its poor plasticity. The primary indication and purpose for LT in patients with UCDs is to prevent neurocognitive impairment (8). Because of the accumulation of neurotoxic metabolites, many argininemia patients may present with increasingly more severe spasticity, seizures or other signs of central nervous system damage during the course of their conservative treatment. Thus, LT is the only available way to prevent neurological deterioration (17). Age of onset, duration of arginine effects, peak levels of plasma arginine, sensitivity of the nervous system to arginine and its metabolites, and genotype may all be related to the outcome, but the underlying mechanisms and associations with these factors require further investigation (4). Moreover, the optimal time for performance of LT in these argininemia patients has yet to be determined. Results from the latest retrospective study involving a large number of cases have found that waitlist duration was associated with a longterm risk for cognitive delay in pediatric LT recipients with UCDs. Accordingly, these findings suggest that an early evaluation for LT should be considered in all children with UCDs to prevent progressive neurologic injury and optimize cognitive outcomes (8). As one example of such benefits, Case 6 received LDLT at an early age to prevent further neurological deficits. She did not suffer severe growth deficiency and walking problems before transplantation, and recovered without any neurological damage until now. Combining our experience with that of previous reports, we recommend that argininemia patients showing a progressive neurological decline following traditional treatments should receive a LT as soon as possible to avoid any further neurological or developmental deficits.
Intellectual developmental disability, irritability, spastic paraplegia as well as other neurological injuries were typical manifestations in our series (1). Results from laboratory assays have also suggested that coagulation dysfunction without bleeding is a characteristic of argininemia (6). While the metabolic decompensation associated with argininemia, such as a clotting disorder and increased levels of arginine, were restored after LT, recovery of existing neurological damage was more problematic (7). A lower limb spasm or contracture is a common long-term complication in argininemia patients, even after LT (7) and treatment of ankle spasms represents a significant challenge in all patients affected by spastic paraplegia. As based on findings from previous reports and our experience, it seems that the more severe the spasm before surgery, the more difficult the recovery. Only after continuous rehabilitation and intermittent botulinum injections to decrease their muscle tone were Cases 7 and 11 able to show a substantial improvement in their ability to walk. As the follow-up time for Cases 7-11 was relatively short, it seems possible that more notable improvements would be observed with future episodes of continuous therapy. In this regard, it is essential to provide neurological and physical rehabilitation for argininemia patients after transplantation through the coordinated efforts among surgeons, physiotherapists, pediatricians, nutritionists and neurologists (25).

In addition to nervous system damage, the effect on the liver was often manifested as slight increases in the levels of ammonia and transaminase. Although the mechanisms of liver injury in argininemia remain unclear, hyperammonemia is considered to be a significant cause of liver damage $(6,26)$. While a coagulation disorder with episodes of hyperammonemia have been described previously, there is no consensus regarding this relationship $(3,6)$. The histopathological results of this group were consistent with those previously reported (27) and were similar to the trends of hepatocellular edema and reversible changes caused by hyperammonemia (28). Combined with imaging and laboratory data, we speculate that liver dysfunction in patients with argininemia may be attributable to hyperammonemia (5).

LT can restore mutated ARG1 enzyme and subsequently provide sufficient enzymatic activity for arginine metabolism. One study found that patients with two severely mutated alleles were indications for LT because rigorous dietary control proved to be relatively ineffective (29). The genetic analysis revealed that all patients were compound heterozygotes, and mutations in six of our patients (Cases 
3-8) were considered severe (29). As shown in animal models, when compared with homozygotes, heterozygotes displayed milder symptoms (18), however, results from other studies have suggested that no significant correlation exists between genotype and phenotype (2). Compared with patients in the DDLT group, those receiving LDLT did not experience sustained elevated arginine levels due to enzyme activity deficiencies after the operation. The data of this study implicate that heterozygotes without clinical symptoms can act as donors for argininemia, however, continuous monitoring is required to identify any potential long-term complications.

Although this was the largest study on LT for argininemia, there are some limitations in our report. Overall, due to the rarity of the disease, it is difficult to conduct a large cohort study and a selection bias may be inevitable as the data for this retrospective study were obtained from a single-center. Advanced and more detailed laboratory analyses, longer follow-up periods, research on the arginine cycle, and establishment of an animal model will help to elucidate the exact mechanisms of argininemia. Future work at our center will be directed toward investigating the effects of developmental delays, protein tolerance, medication and neurological status to assess the long-term efficacy of LT in patients with argininemia.

\section{Conclusions}

LT was an effective treatment for argininemia patients who responded poorly to conservative treatment, which restores arginine metabolism, facilitates physical growth and improves the quality of life in these patients. Based on our experience, we suggest that argininemia patients who have suffered progressive neurological insults should receive LT treatment at an early age at experienced centers, to avoid the development of further neurological or developmental deficits.

\section{Acknowledgments}

We sincerely thank the family members and patients who participated in this study.

Funding: This work was supported by Capital's Funds for Health Improvement and Research (No. 2020-1-2024).

\section{Footnote}

Reporting Checklist: The authors have completed the STROBE reporting checklist Available at https:// tp.amegroups.com/article/view/10.21037/tp-21-576/rc

Data Sharing Statement: Available at https://tp.amegroups. com/article/view/10.21037/tp-21-576/dss

Peer Review File: Available at https://tp.amegroups.com/ article/view/10.21037/tp-21-576/prf

Conflicts of Interest: All authors have completed the ICMJE uniform disclosure form (available at https://tp.amegroups. com/article/view/10.21037/tp-21-576/coif). The authors have no conflicts of interest to declare.

Ethical Statement: The authors are accountable for all aspects of the work in ensuring that questions related to the accuracy or integrity of any part of the work are appropriately investigated and resolved. The study was conducted in accordance with the Declaration of Helsinki (as revised in 2013). This study was approved by the Ethics Committee of Beijing Friendship Hospital, Capital Medical University (No. 2020-P2-094-01). We declare that no organs from executed prisoners were used at Beijing Friendship Hospital, Capital Medical University. All the operations were approved by the Ethical Committee of Beijing Friendship Hospital, Capital Medical University, and all living donors were voluntary and altruistic. Informed consents were obtained from all individual legal guardians of participants included in the study.

Open Access Statement: This is an Open Access article distributed in accordance with the Creative Commons Attribution-NonCommercial-NoDerivs 4.0 International License (CC BY-NC-ND 4.0), which permits the noncommercial replication and distribution of the article with the strict proviso that no changes or edits are made and the original work is properly cited (including links to both the formal publication through the relevant DOI and the license). See: https://creativecommons.org/licenses/by-nc-nd/4.0/.

\section{References}

1. Sin YY, Baron G, Schulze A, et al. Arginase-1 deficiency. J Mol Med (Berl) 2015;93:1287-96.

2. Diez-Fernandez C, Rüfenacht V, Gemperle C, et al. Mutations and common variants in the human arginase 1 (ARG1) gene: Impact on patients, diagnostics, and protein structure considerations. Hum Mutat 2018;39:1029-50.

3. Crombez EA, Cederbaum SD. Hyperargininemia due to 
liver arginase deficiency. Mol Genet Metab 2005;84:243-51.

4. Carvalho DR, Brum JM, Speck-Martins CE, et al. Clinical features and neurologic progression of hyperargininemia. Pediatr Neurol 2012;46:369-74.

5. Scaglia F, Lee B. Clinical, biochemical, and molecular spectrum of hyperargininemia due to arginase I deficiency. Am J Med Genet C Semin Med Genet 2006;142C:113-20.

6. Kiykim E, Zubarioglu T, Cansever MS, et al. Coagulation Disturbances in Patients with Argininemia. Acta Haematol 2018;140:221-5.

7. Silva ES, Cardoso ML, Vilarinho L, et al. Liver transplantation prevents progressive neurological impairment in argininemia. JIMD Rep 2013;11:25-30.

8. Ziogas IA, Wu WK, Matsuoka LK, et al. Liver Transplantation in Children with Urea Cycle Disorders: The Importance of Minimizing Waiting Time. Liver Transpl 2021;27:1799-810.

9. Kasahara M, Sakamoto S, Horikawa R, et al. Living donor liver transplantation for pediatric patients with metabolic disorders: the Japanese multicenter registry. Pediatr Transplant 2014;18:6-15.

10. Morioka D, Kasahara M, Takada Y, et al. Living donor liver transplantation for pediatric patients with inheritable metabolic disorders. Am J Transplant 2005;5:2754-63.

11. Zhu ZJ, Wei L, Qu W, et al. First case of cross-auxiliary double domino donor liver transplantation. World J Gastroenterol 2017;23:7939-44.

12. Jiang YZ, Sun LY, Zhu ZJ, et al. Perioperative characteristics and management of liver transplantation for isolated methylmalonic acidemia-the largest experience in China. Hepatobiliary Surg Nutr 2019;8:470-9.

13. Kossel A, Dakin H. Über die Arginase. Z Physiol Chem. 1904;41:321-31.

14. Cederbaum SD, Yu H, Grody WW, et al. Arginases I and II: do their functions overlap? Mol Genet Metab 2004;81 Suppl 1:S38-44.

15. Ash DE, Scolnick LR, Kanyo ZF, et al. Molecular basis of hyperargininemia: structure-function consequences of mutations in human liver arginase. Mol Genet Metab 1998;64:243-9.

16. Caldwell RB, Toque HA, Narayanan SP, et al. Arginase: an old enzyme with new tricks. Trends Pharmacol Sci 2015;36:395-405.

17. Cui B, Wei L, Zhu ZJ, et al. Neurophysiological characteristics in argininemia: a case report. Transl Pediatr 2021;10:1947-51.

18. Liu XB, Haney JR, Cantero G, et al. Hepatic arginase deficiency fosters dysmyelination during postnatal CNS development. JCI Insight 2019;4:130260.

19. Tang R, Wang X, Zhou J, et al. Defective arginine metabolism impairs mitochondrial homeostasis in Caenorhabditiselegans. J Genet Genomics 2020;47:145-56.

20. Lan M, Tang X, Zhang J, et al. Insights in pathogenesis of multiple sclerosis: nitric oxide may induce mitochondrial dysfunction of oligodendrocytes. Rev Neurosci 2018;29:39-53.

21. Delwing-de Lima D, Wollinger LF, Casagrande AC, et al. Guanidino compounds inhibit acetylcholinesterase and butyrylcholinesterase activities: effect neuroprotector of vitamins E plus C. Int J Dev Neurosci 2010;28:465-73.

22. Amayreh W, Meyer U, Das AM. Treatment of arginase deficiency revisited: guanidinoacetate as a therapeutic target and biomarker for therapeutic monitoring. Dev Med Child Neurol 2014;56:1021-4.

23. Burrage LC, Thistlethwaite L, Stroup BM, et al. Untargeted metabolomic profiling reveals multiple pathway perturbations and new clinical biomarkers in urea cycle disorders. Genet Med 2019;21:1977-86.

24. Miloh T, Barton A, Wheeler J, et al. Immunosuppression in pediatric liver transplant recipients: Unique aspects. Liver Transpl 2017;23:244-56.

25. Sun A, Crombez EA, Wong D. Arginase Deficiency. In: Adam MP, Ardinger HH, Pagon RA, et al. editors. GeneReviews®. Seattle (WA): University of Washington, Seattle; 1993-2022. October 21, 2004.

26. Laemmle A, Gallagher RC, Keogh A, et al. Frequency and Pathophysiology of Acute Liver Failure in Ornithine Transcarbamylase Deficiency (OTCD). PLoS One 2016;11:e0153358.

27. Cederbaum SD, Shaw KN, Spector EB, et al. Hyperargininemia with arginase deficiency. Pediatr Res 1979;13:827-33.

28. Grody WW, Argyle C, Kern RM, et al. Differential expression of the two human arginase genes in hyperargininemia. Enzymatic, pathologic, and molecular analysis. J Clin Invest 1989;83:602-9.

29. Uchino T, Snyderman SE, Lambert M, et al. Molecular basis of phenotypic variation in patients with argininemia. Hum Genet 1995;96:255-60.

Cite this article as: Cui B, Wei L, Sun LY, Qu W, Zeng ZG, Liu Y, Zhu ZJ. The effect of liver transplantation for argininemia - the largest experiences in a single center. Transl Pediatr 2022;11(4):495-504. doi: 10.21037/tp-21-576 
A

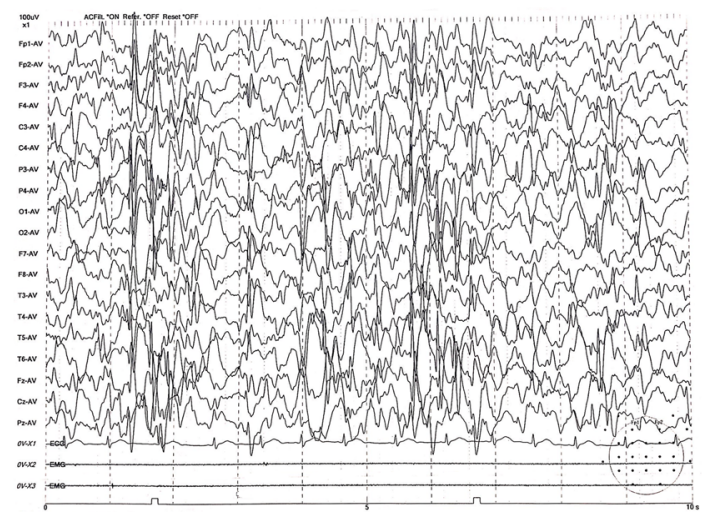

B

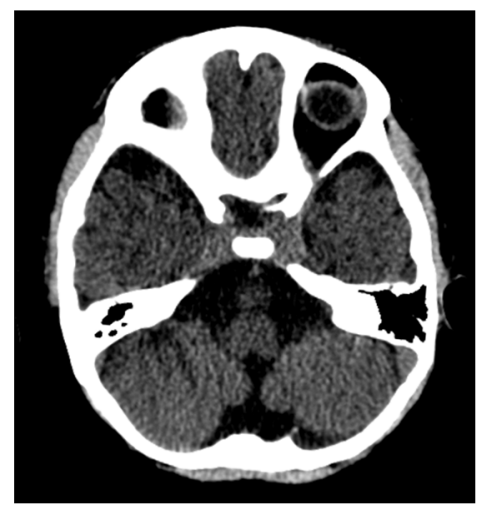

Figure S1 Electroencephalography (EEG) characteristics and neuroimaging of a patient with argininemia. (A) Interictal EEG of Case 10 showed bilateral parieto-occipital, central, and mid-posterior temporal regions are dominated by a continuous asynchrony medium-high amplitude spikes, polyspike/spike-slow complex wave discharges. (B) Neuro-imaging of the Case 11 showed cerebellum, cerebrum and brain stem atrophy.

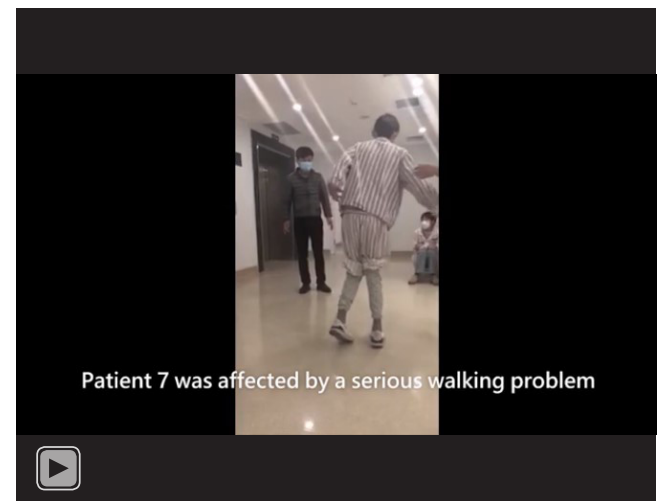

Video S1 The walking posture improved significantly in case 7 after the operation. 